\title{
Cardiovascular Health Risk Assessment and Primary Prevention of Atherosclerotic Cardiovascular Disease
}

\author{
Melvyn Rubenfire ${ }^{1 *}$, Richard L. Weinberg ${ }^{1}$, Sina Jamé ${ }^{1}$, Elif A. Oral ${ }^{2}$, Kenneth A. Jamerson ${ }^{1}$, Robert Brook ${ }^{1}$ \\ ${ }^{1}$ Division of Cardiovascular Medicine, University of Michigan Medical School, Ann Arbor, MI. \\ ${ }^{2}$ Division of Metabolism, Endocrinology and Diabetes, University of Michigan Medical School, Ann Arbor, MI.
}

\begin{abstract}
This review of cardiovascular health risk assessment and therapeutic approach to prevention of atherosclerotic cardiovascular disease (ASCVD) is intended to provide an experienced perspective for health care professionals, and not to replace judgement. Reducing risk factors for ASCVD and related events by prevention affects the most important causes of death and disability in the modern world. Yet ideal cardiovascular health, which is also associated with less cancer, is rare despite the collective efforts of providers, non-profit organizations, governments, employers, the media, and insurance carriers. In fact, there is a worldwide increase in modifiable risk factors.

While optimizing lifestyle is the essential focus for primordial and primary prevention, available generic drugs for the evidence-based prevention treatments are cost effective. And the recent availability of reasonably well calibrated risk estimators (number actual versus number predicted CV events) and recent clinical trials that better inform promotes a more cost/benefit selection process. Herein we review the literature to support the evidence-based rationale and approach to primary prevention of ASCVD from youth to the elderly, discuss the use of risk-estimators and utility of risk enhancers and coronary calcium scores, review guidelines for statin use, aspirin, metabolic syndrome/diabetes, hypertension, and summarize importance of lifestyle.

It's essential a team-based care approach be the strategy to prevent ASCVD that can/should include the primary care physician, nurses, physician extenders, wellness coaches, nutrition and exercise specialists, community health and wellness centers, and referral to prevention specialists as indicated.
\end{abstract}

Keywords: Primary prevention, Atherosclerotic heart disease, Risk estimate, Guidelines.

\section{Introduction}

Cardiovascular disease, in particular atherosclerotic cardiovascular diseases (ASCVD), and contributing risk factors, are the most important causes of death and disability in the developed and many underdeveloped countries in the world [1,2]. Of the nearly 57 million deaths worldwide in 2016, 27\% were due to ischemic heart disease and stroke which have been the leading causes of death globally for many years [1].

The concept of ASCVD prevention has several stages include primordial referring to preventing the development of cardiovascular risk factors (CRF) throughout life in individuals and populations by optimizing lifestyle; primary referring to identifying individuals with CRF with the intention of treating to prevent ASCVD and cardiovascular events (CVE); and secondary referring to strategies to reduce complications, recurrent CVE, and other ASCVD events [3].
That about $25 \%$ of the US population is at risk for ASCVD in the next 10 years underscores the importance and value of identifying those most at risk and implementing the very cost-effective proven strategies [4]. The rationale and key role for screening younger and middle-aged persons for primary prevention is well documented. About 50\% of CV deaths occur in persons without previous symptoms, and $50 \%$ of persons, particularly those with premature ASCVD events, were not aware of or underestimated their risk [5].

Corresponding Author: Dr. Melvyn Rubenfire, 24 Frank Lloyd Wright Dr., Lobby A, Suite 3700, 48106. Received date: June 12, 2020; Accepted date: June 22, 2020; Published date: June 23, 2020.

DOI: https://doi.org/10.31546/2633-7916.1008 


\section{Assessment of ASCVD risk}

An estimate of the 10-year and lifetime risk of heart events and stroke is the foundation of primary prevention that for the individual, including young adults, needs a frank discussion with providers. The enlightened provider helps overcome the bravado of youth and provides each generation the understanding of the importance of a healthy lifestyle, and that treatment of risk factors can prevent premature disability and deaths from heart and vascular diseases, cancer, and all-cause mortality [6].

Experts throughout the globe have developed guidelines for lifestyle, statins, aspirin, and treatment of hypertension which have been shown to reduce ASCVD events in population observational and placebo-controlled trials [7, 8]. Historically, publicity and marketing by pharmaceutical industry using trial results encouraged physicians to prescribe expensive medication with little to no long-term evidence of value, and an inability to decide who would benefit. While there was evidence of relative risk reduction of events from aspirin, statins, and anti-hypertensive treatments, the number needed to treat (NNT) or harm $(\mathrm{NNH})$ to prevent an event and cost/benefit was not known, particularly for sub-groups. More recently, US, European, and World Health Organization experts developed guidelines and encouraged use of electronic and paper chart risk estimators utilizing population observational studies, and clinical trial outcomes with the goal of matching need and intensity of preventive therapies to absolute risk (generally $10 \mathrm{yr}$ and lifetime) for ASCVD events [8,9]. The free and easily accessible online tools for estimating cardiovascular outcomes provides the opportunity to select the risk estimator that fits the patient by variables that include age, sex, ethnicity, country, socioeconomic status, and high versus low risk. The most recent ACC/AHA US iteration, http://tools.acc.org/ascvd-risk-estimator-plus [9], provides an estimate of $10 \mathrm{yr}$ or lifetime risk of ASCVD (including fatal and non-fatal), defined as acute coronary syndromes, myocardial infarction, stable or unstable angina, arterial revascularization, stroke/transient ischemic attack, peripheral arterial disease, and includes expected risk reduction based upon clinical trials of statins, antihypertension drugs, and aspirin [10]. It is both useful for the clinician to assess risk and inform the patient on the value of treatments that include estimate of risk reduction. The European Heart Score (SCORE, http://www.heartscore.org was developed to estimate the $10 \mathrm{yr}$ cumulative risk of a fatal ASCVD event in persons up to 65 yrs [11]. Total CVD event risk is about $3 \mathrm{x}$ higher than risk for fatal CVD events.

\section{Deciding treatment options and indications for prevention of ASCVD}

There is a highly significant difference in guideline recommendations between US and low risk European countries. In Europe, a 65yr old man with BP $140 \mathrm{mmHg}$, total cholesterol $220 \mathrm{mg} / \mathrm{dL}$, and HDL-C 42 has a $5 \% 10 \mathrm{yr}$ risk of a CV death using Q-RISK3.
As $5 \%$ is defined as low risk in the guideline, recommendation is limited to diet and exercise. The same patient in the U.S. has a $17 \% 10 \mathrm{yr}$ risk of an ASCVD event using ACC/AHA ASCVD risk+ (intermediate risk), and is recommended a statin, antihypertensive drug to lower the sBP to $<130 \mathrm{mmHg}$ and low dose aspirin which together lowers the $10 \mathrm{yr}$ risk of ASCVD events to $8.8 \%$. Of course, the recommendation for aspirin in patients at moderate risk of CVD was not supported in the most recent trial [12]. Amongst the limitations of the risk estimator/treatment guideline tools is the difficulty of updating evidence-based treatments.

The US ACC/AHA guideline estimates 10yr ASCVD risk (AR10) for asymptomatic adults 40-79yrs. Categories are low <5\%, borderline $5-7.4 \%$, intermediate $\geq 7.5 \%$ to $<20 \%$, and high risk $\geq 20 \%$ [10]. The accuracy of the estimated risk applies to persons with the risk factors assessed but limited in those whose risk may be higher or lower depending on many unmeasured variables such as lifelong lifestyle, education, race/ethnicity, social determinants, metabolic risk factors, and renal function. The US guidelines suggest using riskenhancers to decide initiation or intensification of statin therapy in patients who are borderline or intermediate risk (Figure 1) [10]. While important to supplement judgement the risk estimators are far from perfect. However, when the risk calculator is utilized and shared with the patients, more guideline adherence is observed [13]. Guidelines with risk estimates provide dosing of statins and under dosing statins has significant consequences. In a prospective study of primary care patients without ASCVD, using a guideline of $>40 \%$ reduction in LDL-C from baseline as optimal, over half had a sub-optimal response [14]. Compared to optimal responders, subsequent incident CVD for sub-optimal responders was $22 \%$ higher (adjusted for baseline untreated LDLC).

2018 US cholesterol guidelines incorporate other
risk factors and markers, enhanced estimates, and
'the lower is better hypothesis'
The 2018 ACC/AHA cholesterol guideline The 2018 ACC/AHA cholesterol guideline
emphasizes reducing risk of ASCVD through lipid management for persons at risk and with established ASCVD [10]. It emphasizes a more intensive personalized approach based upon recent controlled studies: in high-risk patients the lower the LDL-C, regardless of treatment type, the better the outcome. It includes a novel risk-estimator for primary prevention that can be used for decisions regarding statins, hypertension and aspirin use and incorporates personalized treatment options (to be discussed). 
Table 1 is a modified version of the key perspectives from the 2018 ACC/AHA guideline [10] focusing on primary prevention with suggestions added in italics.

Table 1: Cholesterol guidelines incorporate other risk factors and markers, enhanced estimates, and 'the lower is better hypothesis' (as modified from reference10).

1. Emphasize a heart-healthy lifestyle across the life course. In adults 20-39 years of age, an assessment of lifetime risk as described below facilitates the clinician-patient risk discussion and emphasizes intensive lifestyle efforts.

2. In severe primary hypercholesterolemia (LDL-C level $\geq 190 \mathrm{mg} / \mathrm{d}$ ) begin high-intensity statin therapy. If the LDL-C remains $\geq 100 \mathrm{mg} / \mathrm{dL}$, adding ezetimibe is reasonable. Patients with heterozygous familial hypercholesterolemia (HeFH) can be prescribed PCSK-9 inhibitor therapy regardless of presence of ASCVD because of very high-risk. Those with HeFH and an LDL-C of $190 \mathrm{mg} / \mathrm{dl}$ have a 3-4-fold greater risk of CV events than others at the same LDL-C level and 20-fold greater risk than those with an LDL-C of $130 \mathrm{mg} / \mathrm{dl}[16]$.

3. Diabetics 40 to 75 years of age and an LDL-C level of $\geq 70 \mathrm{mg} / \mathrm{dL}$, begin moderate-intensity statins without calculating 10-year ASCVD risk. It is reasonable to use a high-intensity statin to reduce the LDL$\mathrm{C}$ by at least $50 \%$ in patients with T2DM with diabetes risk enhancers (T2DM 10 years and T1DM for 20 years or longer duration, albuminuria $\geq 30 \mathrm{mcg} / \mathrm{mgCr}$, retinopathy, $\mathrm{ABI}<0.9$, eGFR $<60 \mathrm{ml} / \mathrm{min} / 1.73 \mathrm{~m}^{2}$, neuropathy), especially those with multiple CV risk factors or those 50 to 75 years of age [10].

4. Adults 40-75 years of age evaluated for primary ASCVD prevention, have a clinician-patient risk discussion before starting statin therapy. Risk discussion should include a review of major risk factors and calculated 10-year risk of ASCVD; the presence of risk-enhancing factors (Figure 1). Patient preferences and values in shared decision-making should guide the decision.

5. In adults 40 to 75 years of age without diabetes mellitus and with LDL-C levels $\geq 70-189 \mathrm{mg} / \mathrm{dL}$, at a 10 year ASCVD risk of $\geq 7.5 \%$ start a moderate-intensity statin if a discussion of treatment options favors statin therapy. Risk-enhancing factors favor statin therapy and consideration of increasing dosing (Figure 1). If risk status is uncertain, consider a coronary artery calcium score (CACs) as discussed below. If the 10-year risk is $\geq 20 \%$, reduce LDL-C by $\geq 50 \%$. If statins are indicated based upon intermediate risk, reduce LDL-C levels by $35-50 \% \%$. When compared to $10 \mathrm{mg}$ of rosuvastatin in a primary prevention study population at intermediate risk [17], high-intensity $(20 \mathrm{mg})$ rosuvastatin in the JUPITER trial [18] achieved greater reduction in $L D L-C$ and greater reduction in CV events.

6. In adults 40 to $\mathbf{7 5}$ years of age without diabetes mellitus and 10 -year risk of 5\%-19.9\%, risk-enhancing factors favor initiation and consideration of intensification of statin therapy (Figure 1). Other risk enhancing factors include radiation therapy for left breast cancer and other cancers such as lymphoma when the left main, left anterior descending, and proximal right coronary artery is in the field of treatment [19], and post-traumatic stress disorder [20].

7. In adults 40 to 75 years of age without diabetes mellitus and with $L D L-C \geq 70 \mathrm{mg} / \mathrm{dL}-189 \mathrm{mg} / \mathrm{dL}$, and a 10-year ASCVD risk of $\geq 5 \%-19.9 \%$, if a decision about statin therapy is uncertain, consider measuring CAC. If the coronary artery calcium score (CAC) is zero, treatment with statin therapy may be withheld or delayed, except in cigarette smokers, diabetics, and those with a strong family history of premature ASCVD. A $\mathrm{CAC}$ score of $>0$ favors statin therapy in $\mathrm{HeFH}$ and in young persons. For any patient, if the CAC score is $\geq$ 100 Agatston units or $\geq 75$ th percentile, statin therapy is indicated unless otherwise deferred by the outcome of clinician-patient risk discussion.

8. Assess adherence and percentage response to LDL-C-lowering medications and lifestyle changes with repeat lipid measurement 4 to 12 weeks after statin initiation or dose adjustment, repeated every 3 to 12 months as needed. Define responses to lifestyle and statin therapy by percentage reductions in LDL$\mathrm{C}$ levels compared with baseline. In patients at very high-risk, triggers for adding non statin drugs are defined by threshold LDL-C $>70 \mathrm{mg} / \mathrm{dL}$ on maximal statin therapy. 


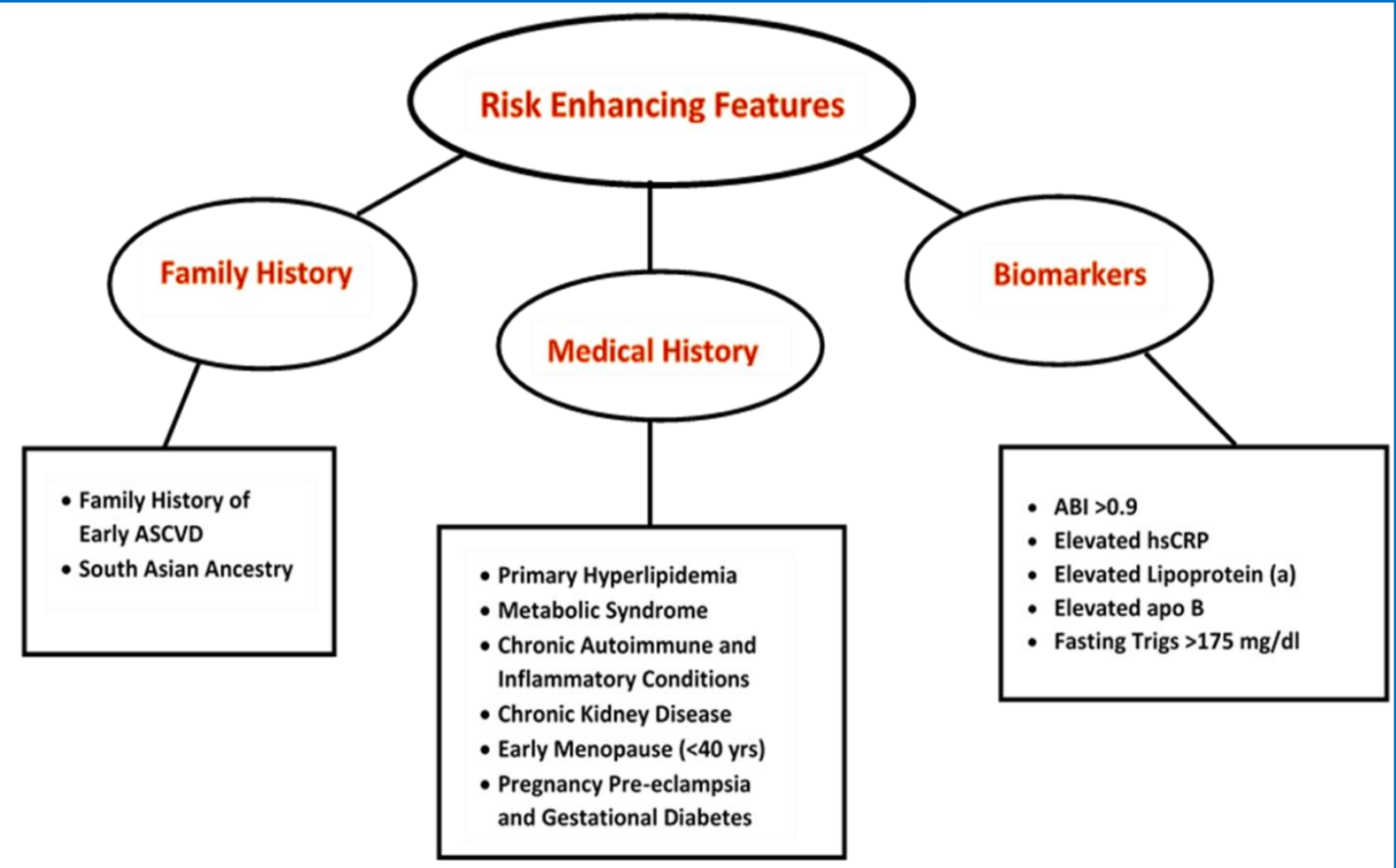

Early ASCVD - men <55, women <65; High cholesterol (LDL-C 160-189 mg/dL; non-HDL-C 190-219 mg/dL); Inflammatory conditions (RA, psoriasis, SLE, HIV); Elevated lipoprotein (a) ( $\geq 50 \mathrm{mg} / \mathrm{dL}$ or $\geq 125 \mathrm{nmol} / \mathrm{L}$, Elevated apolipoprotein $B(\geq 130$ $\mathrm{mg} / \mathrm{dL}$. ABI - ankle brachial index.

Figure 1: Summarizes the risk enhancers.

\section{Cost-effectiveness of Statins}

There is evidence for cost-saving by statins in CHD which improves with intensifying dosing as well as adding additional lipid lowering drugs such as ezetimibe [20,21]. Statin therapy for primary prevention of ASCVD using the ACC/AHA cholesterol guidelines with $10 \mathrm{yr}$ threshold of $7.5 \%\left(\mathrm{AR}_{10}\right)$, the threshold for intermediate risk, has been shown to be cost effective [22], but initial calculations have not considered those with higher LDL-C have greater absolute reduction in events from statins and may be costsaving. A recent simulation study assessed the costeffectiveness of expanding statin treatment eligibility in men and women age 40 years at baseline from standard threshold of $7.5 \%$ to $\mathrm{AR}_{10} 5.0 \%$ to $7.4 \%$ (definition of borderline risk) [23]. Treating with a statin for individuals with borderline $\mathrm{AR}_{10}$ and LDL-C levels of 160 to 189 $\mathrm{mg} / \mathrm{dL}$ would be cost-saving; treating borderline $\mathrm{AR}_{10}$ and LDL-C levels of 130 to $159 \mathrm{mg} / \mathrm{dL}$ would also be costsaving; and treating all individuals with $\mathrm{AR}_{10}$ of at least $5.0 \%$ would be highly cost-effective (\$33,558/QALY) and of course prevent the most ASCVD events. Costeffectiveness increases with LDL-C level and $\mathrm{AR}_{10}$ [23]. In view of the low cost and no to minimal evidence of longterm harm, consideration should be given to statin therapy for the borderline risk group of $5 \%$ to $7.4 \% \mathrm{AR}_{10}$ with LDL-C >129mg/dL, and possibly lower LDL-C particularly in the presence of risk enhancers (Figure 1).

\section{Statins for the Elderly}

Treatment strategies for those over 60yrs (statin, aspirin, BP) are less clear because of the limited number of elderly in population and RCT's from which to derive risk estimate [24]. The decision algorithm regarding statins should not change in the elderly who have a life expectancy of at least 5 years. In a recent meta-analysis of randomized placebocontrolled statin trials, there was a $21 \%$ proportional reduction in major CVE per $\sim 40 \mathrm{mg} / \mathrm{dL}$ reduction in LDL-C, which decreased insignificantly with age, but was significant in all age groups [25] Statins and more intensive statins are associated with $25 \%$ reduction in proportional risk of coronary revascularization and a $16 \%$ reduction in risk of stroke which does not differ across age groups. The benefit of statins is similar for both men and women and persons over 75 yrs [26,27]. Further, at an average of 2.4yrs of follow-up of elderly on statins, the $14 \%$ who discontinued statins for at least $3 \mathrm{mo}$ had $33 \%$ more CV events $(46 \%$ coronary and $26 \%$ cerebrovascular) [27]. 
Statins are safe in the elderly and have no effect at any age on non-vascular mortality, cancer death, or cancer incidence. Given the aging population and lack of evidence from randomized controlled trials in people $>75$ years, physicians and their patients must use shared decisionmaking on when to use statins for primary prevention. Particularly considering that simply by the age of $70 \mathrm{yrs}$, women have an 8\% 10-year risk for ASCVD events. Absence of informed data should not be confused with lack of effect given the high absolute risk and very low cost of statins that reduce stroke risk.

\section{Statins in younger persons}

The 2018 AHA/ACC cholesterol guidelines for men and women 20-39 years emphasize a healthy lifestyle for all, as well as estimating the life-time risk which can be done with the ASCVD Risk Estimator Plus [9] or for 30 years and above with the QRISK-lifetime cardiovascular risk calculator [28]. QRISK lifetime was developed in the UK primary care population and reasonably approximates the US. Low risk for survival to age 95 years is 5\% and high risk is over $39 \%$. Statins should be considered for those 20 39 years if there is a family history of premature ASCVD and LDL-C >160mg/dL; a high lifetime risk estimate; diabetes or elevated $\mathrm{Lp}$ (a) with another major risk factor; or a CAC score $>0$ [10].

Enhancing ASCVD Risk Estimates with Quantitative Tools After using the tools for ASCVD risk estimate, clinical judgment should be based on individual patient's preferences, and presence of other risk enhancers. Adding these variables and selective use of coronary artery calcium scoring (CAC score) can help to overcome most issues of mis-calibration for each of the many risk prediction equations [29]. The use of CAC scoring by chest computed tomography has increased with the increase in evidence of value and decreasing fees. It can be obtained without a physician order in some states in the US, is generally not covered by insurance, and fee can range from $\$ 75$ to $\$ 250$. Scoring techniques are well standardized and provide a reproducible means of assessing risk of major cardiovascular outcomes. CAC testing in asymptomatic populations is cost-effective through a broad range of baseline risk [30]. Further, the CAC score identifies persons most likely to benefit from statins. The number needed to treat to prevent one MACE outcome over 10 years ranges from 100 for CAC 1-100 to 12 with CAC > 100 [31].

The Multi-Ethnic Study of Atherosclerosis (MESA) study confirmed that coronary calcium scoring by computed tomography can predict CHD events independent of classic risk factors and novel risk markers [32,33]. Despite the complexity of atherosclerotic plaque that results in CHD events, the predictive value of increasing CAC provides an estimate of both hard and soft non-calcified so called 'vulnerable plaque'[34-36]. A CAC score $>100$ has a 7-fold increase in CHD event rate compared to $\mathrm{CAC}=0$ [36].
The value of high CAC for predicting major cardiovascular events (MACE) and the value of statins has been well established, but the 'Power of $\mathrm{CAC}=0$ ' is equally, and to some extent, more valuable [36]. There is a clear difference between risk with a $\mathrm{CAC}=$ 0 and CAC range 1-10, particularly in young persons. The 2018 guideline on cholesterol management suggests a CAC scan is indicated when the decision for statin therapy is uncertain. This may include patients who appear to be at intermediate or higher risk but remain skeptical; those at low risk $(<5 \%)$ based upon age but with a family history of premature CHD; an isolated CV risk factor such as low HDL-C, LDL-C $>160 \mathrm{mg} / \mathrm{dL}$, or high Lp(a), and importantly those with statin intolerance [10].

A risk estimator using the MESA data was developed to incorporate the CAC score and was validated in 2 other cohorts [37]. The risk estimate combines the CAC score with standard risk factors and prevention treatment at the time of the CAC study. MESA Risk Estimate improves discrimination for CHD events including myocardial infarction, angina if followed by revascularization, resuscitated cardiac arrest, and CHD death, but does not include stroke. However, in population studies with a relatively low stroke risk (0.3\% per year) CAC score has a strong relationship with ischemic stroke independent of the LDL-C, (range CAC $=00.1 \% / \mathrm{yr}$ to $\mathrm{CAC} \geq 4000.70 \% / \mathrm{yr}$ [38]. Use of the MESA risk score and treatment alone, can influence patient decisions and enhance compliance with treatment.

https://mesanhlbi.org/MESACHDRisk/MesaRiskScore /RiskScore.aspx

\section{A summary of recommendations for interpretation} of CAC score is as follows [10, 30, 36].

1. $\quad \mathrm{CAC}=0$ : Most often below threshold for statin benefit. Consider avoiding or postponing drug therapy with exception of diabetics, current heavy smokers, or a strong family history of premature ASCVD. Can be used to delay therapy in heterozygous familial hypercholesterolemia $(\mathrm{HeFH})$, particularly in women of child baring age who are not using any birth control methods. If statins are held because of a CAC $=0$, the CAC score should be repeated in 4-5 years.

2. $\mathrm{CAC} \geq 100$ or $>75$ th percentile for age/sex/race: Recommend statin therapy, intermediate or high intensity.

3. CAC 1-99 and <75th percentile score for age/sex/race: Subclinical atherosclerosis is present; use MESA risk-estimate with CAC score as a continuous variable. Use a statin if estimate is $7.5 \%$ or greater and consider statin with score $5 \%$ $7.4 \%$ if there are risk enhancers. 
4. Consider statins for men and women with a CAC $>0$, particularly if 20-39 years old. Repeat discussion with patient if there is new information. If statin is postponed based upon CAC, consider repeating CAC score in 4-5 years.

\section{Aspirin for primary prevention}

The results of 3 recent pivotal trials did not support low dose aspirin even for what would intuitively be high risk patients, including the healthy elderly [39], those at moderate risk for CVD [12], and diabetics [40]. A subsequent systematic review and meta-analysis of RCT's included the three recent trials [41]. A total of 13 trials had 164,225 participants with $>1$ million participant-years of follow-up; average age 62yrs (range 53-74), 47\% men, 19\% had diabetes, and the median baseline $10 \mathrm{yr}$ risk of the primary CV outcome was $9.2 \%$ (ranges $2.6 \%-15.9 \%$ ).

It was concluded that aspirin in persons without CVD was associated with a lower risk of CVE but with an increased risk of major bleeding. The absolute risk reduction was $0.38 \%$ (NNT 265) and absolute increase in major bleeding (mostly gastrointestinal) of $0.47 \%$ (NNH 210) [42]. However, taking the data in its totality, low dose aspirin could be considered in specific cohorts with low risk for GI bleeding and intracranial hemorrhage (<70yrs for each) who are on statins with good BP control and at least a $10 \%$ $\mathrm{AR}_{10}$ for $\mathrm{CV}$ events based upon the ACC/AHA risk estimate, active smokers, and possibly lipoprotein (a) $>50 \mathrm{mg} / \mathrm{dL}[12,41,42]$.

\section{Lifestyle intervention}

The value of physician provided advice for a healthy lifestyle, even in persons who do not appear at risk, should not be underestimated. That physicians impact patient behavior is the rationale for the US Preventive Service Task Force recommending patient education as part of routine care [43]. The most common and readily detectable risk factors amenable to primary prevention for ASCVD as well as all-cause and cancer mortality in young and middle-aged persons occur in clusters and are each interactive with lifestyle [2].

These factors include smoking; hypertension; obesity; physical inactivity; high risk lipids; pre-diabetes, diabetes, and the metabolic syndrome; psychological distress and depression, and poor oral health [44-51]. The benefit of exercise, exercise intensity, and aerobic cardiorespiratory fitness on long-term mortality is particularly impressive in those over 70yrs and with hypertension [52]. The amount of time older women spent doing light physical activity (similar to leisurely walking) is independently associated with a lower likelihood of CVE.
Each additional hour-per-day increment in light physical activity is associated with a $10 \%$ lower risk of CVE. The incremental risk for reduced fitness is comparable to or greater than the presence of CHD, diabetes, and smoking and provides further support for achieving aerobic fitness as a modifiable risk factor for long-term mortality [52].

Amongst the reasons for the very high risk of ASCVD in poor countries and poor cities and rural areas in all countries is related to lack of health care providers, appropriate medication/education, healthy foods, and smoking. The recent HOPE-4 study demonstrated that a low-cost community-based identification and treatment of hypertension and other $\mathrm{CV}$ risk factors can be very effective in populations with little knowledge and low socioeconomic status [53]. There are no reasons why similar programs could not be implemented in urban and rural communities in need in the US and other developed and underdeveloped countries.

\section{Approach to hypertension}

Hypertension (HTN) is the most important treatable risk factor responsible for the sum of major CVE including acute coronary syndromes, heart failure, atrial fibrillation, strokes, and CV deaths [54]. The overall prevalence of HTN defined as $\mathrm{sBP} / \mathrm{dBP} \geq$ $130 / 80 \mathrm{mmHg}$ in the US is $46 \%$ and for men and women $65-74 y r s$ it is about $75 \%$. The 2017 ACC/AHA Guideline for defining, testing, and treating HTN is summarized in Table-2 [55].

It stresses the importance of out of office measures as home and 24-hour ambulatory values better predict CVE and facilitate screening for white coat (high BP in office and normal at home) and masked hypertension (normal BP in the office and elevated BP at home). Emphasis is on life-style modification with the DASH-Mediterranean diet, exercise, weight control, and the importance of screening for and managing other CVD risk factors. Use of BP lowering medication is recommended for primary prevention in adults with an estimated $\mathrm{AR}_{10} \geq 10 \%$ and $\mathrm{sBP} \geq 130 \mathrm{~mm} \mathrm{Hg}$ or $\mathrm{dBP} \geq 80 \mathrm{~mm} \mathrm{Hg}$ (http://tools.acc.org/ascvd-risk-estimator-

plus/\#!/calculate/estimate/). Use of BP-lowering medication is also recommended in adults with an estimated $\mathrm{AR}_{10}<10 \%$ and $\mathrm{sBP} \geq 140 \mathrm{~mm} \mathrm{Hg}$ or a $\mathrm{dBP}$ $\geq 90 \mathrm{~mm} \mathrm{Hg}$. The recommended goal for BP is $<130 / 80 \mathrm{mmHg}$ for primary prevention, diabetes, chronic kidney disease, and ASCVD. 
Table 2: Defining, testing, treatment considerations in hypertension.

- Defining hypertension

- Normal sBP $<120$ and $\mathrm{sBP}<80$

- Elevated sBP 120-129 and $\mathrm{dBP}<80$

- Hypertension stage 1 sBP $130-139$ or dBP $80-89$

- Hypertension stage $2 \mathrm{sBP} \geq 140$ or $\mathrm{dBP} \geq 90$

- Prior to labeling a person with hypertension: use an average based on $\geq 2$ readings obtained on $\geq$ 2 occasions to estimate the individual's BP.

- Screen for white coat or masked HTN: home BP (HBP) or $24 \mathrm{hr}$ ambulatory BP monitoring (AMBP)

- Clinic $130 / 85=$ HBP $130 / 80=$ daytime AMBP $130 / 80$

- Clinic $140 / 90=$ HBP $135 / 85=$ daytime AMBP $135 / 85$

- Clinic 160/90 = HBP 145/90 = daytime AMBP 145/90

- Drug resistant HTN $=$ BP above goal despite maximal dosing of 3 anti-hypertensive drugs.

- Resistant hypertension: BP at goal but requiring 4 or more drugs

- Screen and treat CV risk factors: smoking, diabetes, dyslipidemia, excessive weight, low fitness, unhealthy diet, psychosocial stress

- Screen for: drug-induced nonsteroidal anti-inflammatory, steroids, androgens, decongestants, caffeine, cocaine, monoamine oxidase inhibitors, alcohol.

- Basic testing: CBC, FBS, lipids, basic metabolic panel, TSH, urinalysis, electrocardiogram with optional echocardiogram, uric acid, and urinary albumin-to-creatinine ratio.

- Screening for secondary causes: new-onset or uncontrolled HTN, drug-resistant $(\geq 3$ drugs at maximal dosing), abrupt onset, age $<30$ years, excessive target organ damage (cerebral vascular disease, retinopathy, LVH by echo or ECG, HFpEF, HFrEF, CAD, CKD, peripheral artery disease, albuminuria), onset of diastolic hypertension in older adults, unprovoked or excessive hypokalemia.

- Test for common secondary causes: renal parenchymal disease, renovascular disease, primary aldosteronism, obstructive sleep apnea.

- Test if more specific clinical characteristics are present: neuroendocrine tumor, Cushing's syndrome, congenital adrenal hyperplasia, primary hyperparathyroidism, and aortic coarctation.

- All patients should be educated regarding optimal weight, DASH-Mediterranean diet, and exercise. Prior to drug therapy all patients with elevated BP, stage $1 \mathrm{HTN}$ and younger persons with stage $2 \mathrm{HTN}$ should undergo $3-6 \mathrm{mo}$ of evaluation and lifestyle intervention. Initial first-line drug therapy for stage 1 HTN includes the diuretic chlorthalidone $12.5-25 \mathrm{mg}$, a calcium channel blocker preferably amlodipine, or ACEi or ARB. Stage $2 \mathrm{HTN}$ is best treated with 2 first line drugs, preferably in combination for once daily dosing (e.g. ACEi or ARB + CCB or ACEi or ARB + diuretic). Adults with a very high average BP (e.g. $\geq 160 \mathrm{~mm} \mathrm{Hg}$ or $\mathrm{DBP} \geq 100 \mathrm{~mm} \mathrm{Hg}$ ) require prompt evaluation and drug treatment followed by careful monitoring and upward dose adjustment to target to less than $130 / 80 \mathrm{mmHg}$. Beta blockers are indicated for hypertension only when indicated in CAD with angina or ischemia, HFrEF, and arrhythmias.

- The addition of spironolactone or eplerenone are indicted for drug resistant hypertension.

Abbreviations: SBP (systolic blood pressure), dBP (diastolic blood pressure), CV (cardiovascular), LVH (left ventricular hypertrophy), HFpEF (heart failure preserved systolic function), HFrEF (heart failure reduced systolic function), CAD (coronary artery disease), CKD (chronic kidney disease), ACEi (angiotensin-converting-enzyme inhibitor), ARB (angiotensin II receptor blocker).

\section{Obesity, metabolic syndrome, and type 2 diabetes mellitus (T2DM)}

Obesity is defined by body mass index (BMI) $\geq 30 \mathrm{~kg} / \mathrm{m} 2$ in U.S. Caucasians and African Americans, and $\geq 25 \mathrm{~kg} / \mathrm{m} 2$ in Asians, Middle Eastern, and Mediterranean cohorts. It tends to reflect family lifestyle and genetics and independently increases risk for dyslipidemia, impaired fasting blood glucose or pre-diabetes (FBG $100-125 \mathrm{mg} / \mathrm{dL}$, A1c 5.7-6.4\%), type 2 diabetes mellitus (T2DM, A1c $\geq$ $6.5 \%$ ), and hypertension [55]. The metabolic syndrome, which is associated with a fasting serum insulin disproportionate to the FBG (insulin resistance) has an - approximate 2-fold lifetime risk of diabetes and ASCVD. Risk factors associated with insulin resistance include dyslipidemia with increased triglycerides and decreased HDL-C, small atherogenic LDL particles and increased atherogenic VLDL remnant particles, hypertension, hyperglycemia, reduced fibrinolysis, increased inflammation, and endothelial dysfunction. It is inherited, increases with age, obesity, and sedentary lifestyle, results from polycystic ovary syndrome, and often causes or may be precipitated by non-alcoholic fatty liver disease. [56]. 
The definition of the metabolic syndrome in the U.S. includes 3 of the following 5 criteria: $\mathrm{BP} \geq 130 / 85 \mathrm{mmHg}$ or on anti-hypertensive medication, impaired fasting glucose, diabetes or use of hypoglycemic drugs, triglycerides (trig) $>150 \mathrm{mg} / \mathrm{dL}$ or on trig lowering drugs, HDL-C in men $<40 \mathrm{mg} / \mathrm{dL}$ and in women $<50 \mathrm{mg} / \mathrm{dL}$, and abdominal obesity defined as $\geq 40$ " in men and $\geq 35$ " in women. Normal weight or mildly overweight metabolic syndrome is not uncommon and important to consider for prevention of T2DM and ASCVD. In contrast, metabolically healthy obesity and the obesity paradox of improved survival from major cardiovascular events have been well described [57].

Recommendations for obesity, metabolic syndrome, and diabetes without ASCVD as modified from guidelines are reviewed in Table-3 $[55,56,57]$. Treatments delay the onset of diabetes and microvascular complications. Most importantly, moderate weight loss $(\geq 5 \%)$ in the year following the diagnosis of T2DM is associated with improvement in risk factors and a $48 \%$ lower hazard of CVD at 10 years [57]. While there is documented benefit of physician referral to multidisciplinary diet and exercise programs designed to improve the components of the metabolic syndrome, there is no evidence for persistence of benefit or a legacy effect [58].
The choice of agents for diabetes varies considerably among experts and is highly dependent on insurance and cost. The introduction of the sodium-glucose cotransporter 2 (SGLT-2) inhibitors and glucagon-like protein 1 (GLP-1) receptor agonists has created a new paradigm for treating T2DM, particularly with ASCVD, CKD, CHF, hypertension, and obesity [56]. Clinical trials in progress will inform their costeffectiveness in diabetes at high risk for ASCVD.

Another breakthrough in diabetes management was reported in REDUCE-IT, a randomized placebocontrolled trial of icosapent ethyl (Vascepa ${ }^{\mathrm{TM}}$ a synthetic eicosapentaenoic acid) [59]. Participants with ASCVD or diabetes with at least 1 risk factor and fasting triglycerides $135 \mathrm{mg}$ to $500 \mathrm{mg} / \mathrm{dL}$ (mean $216 \mathrm{mg} / \mathrm{dL}$ ) on an appropriate dose of a statin (on trial baseline LDL-C $75 \mathrm{mg} / \mathrm{dL}$ ) were randomized to 2 grams twice daily of icosapent ethyl or matching placebo. Nearly $30 \%$ enrolled on the basis of primary prevention in diabetes. There was a $25 \%$ reduction in the primary endpoint of combination of cardiovascular deaths, non-fatal MI, non-fatal stroke, coronary vascularization or unstable angina. The benefit did not correlate with baseline triglycerides or reduction of triglycerides suggesting non-lipid benefits of icosapent ethyl.

Table 3: Obesity, metabolic syndrome, and type 2 diabetes mellitus (T2DM) (as modified 55, 56 \& 57).

- Appropriate energy limited weight management diet with initial weight loss goal of $5 \%$ in $3 \mathrm{mo}$ and $10 \%$ in $6 \mathrm{mo}$.

- Diet high in fiber with low glycemic index and low saturated fat; e.g. Mediterranean, DASH, or pescavegetarian. Avoidance of fad diets without evidence for long term benefit

- Moderate physical activity $150 \mathrm{~min} /$ week minimum, 200-400min/week for weight loss, 200min/week to prevent weight gain after loss.

- $\quad$ Target BP to $<130 / 80 \mathrm{mmHg}$ with diet, exercise, and drugs as per Table 1 .

- In diabetes with one or more CV risk factors or ASCVD with triglycerides $>135 \mathrm{mg} / \mathrm{dl}-500 \mathrm{mg} / \mathrm{dl}$, consider high dose icosapent ethyl (synthetic EPA)

- Glucose control target Alc to $6.5-<7 \%$ with care to avoid hypoglycemia and monitor renal function with dose adjustment or avoidance of certain drugs with eGFR $<45 \mathrm{~mL} / \mathrm{min}$

- Metabolic syndrome or pre-diabetes: if inadequate improvement in glycemic control and weight loss with diet and exercise, after 3 months consider metformin

- Diabetes: For A1c 6.5 to $7.5 \%$ begin with lifestyle interventions. If not at goal or if at onset of diagnosis A1c $>7.5 \%$ initial lifestyle program with

- First line drug metformin and $2^{\text {nd }}$ thiazolidinediones (TZD's, preferably pioglitazone), avoid sulfonylurea (SU) if possible. If cost is prohibitive, consider the long acting SU glimepiride which does not increase $\mathrm{CV}$ events rate

- If compelling need for weight loss, sodium -glucose cotransporter inhibitor (SGLT2 inhibitors) and glucagon-like peptide-1 receptor agonist (GLP-1 agonist) are uniquely capable of reducing CVD events

- Prior to insulin, consider the dipeptidyl peptidase IV inhibitors ('gliptins').

- For very high baseline A1c (e.g. $>8.5-9 \%$ ), begin with GLP-1 agonist. If A1c is $>9 \%$ or FBS $>250 \mathrm{mg} / \mathrm{dl}$ with ketonuria or weight loss, insulin is indicated. If no weight loss, can begin with GLP-1 agonist.

- The clinical A1c should be checked at least $2 \mathrm{x}$ per year and $4 \mathrm{x}$ for persons with highly variable results, or microvascular complications. The degree of self-monitoring is highly patient dependent. For those with an A1c $<7 \%$, it should be about weekly and anytime there are symptoms suspicious of hypoglycemia.

- Assess yearly for moderately increased albuminuria defined as spot urine albumin to creatinine ratio of 30 and $300 \mathrm{mg} / \mathrm{g}$ of creatinine which is similar to $30-300 \mathrm{mg}$ albumin per day. Glycemic and BP control as well as ACEi and ARBs reduce albuminuria and the risk of development of diabetic nephropathy 


\section{Acknowledgements: None}

\section{Authorship Statement}

Principle author, conceptual design, responsible for writing and editing: (MR). Contributed manuscript materials and sections: (RLW, RB). Preparation of figure and tables, manuscript review: (JS). Contribution of diabetes and obesity information: (EAO). Contribution of hypertension information: (RB, KJ). Review and approval of final manuscript version: all authors.

\section{Funding}

This research did not receive any specific grant from funding agencies in the public, commercial, or not-forprofit sectors.

\section{Conflict of Interest}

The authors have no conflicts of interest to declare. Kenneth Jamerson, MD is a paid consultant for Cunjupro Bio-Pharmaceutical and ReCor Pharma.

\section{References}

1. World Health Organization. The top 10 causes of death: Global Health Estimates 2016: Deaths by Country and Region, 2000-2016. Geneva: World Health Organization: 2018.

2. GBD 2015 Risk Factors Collaborators. Global, regional, and national comparative risk assessment of 79 behavioural, environmental and occupational, and metabolic risks or clusters of risks, 1990-2015: a systematic analysis for the Global Burden of Disease Study 2015. Lancet. 2016;388:1659-1724.

3. Turco JV, Inal-Veith A, Fuster V. Cardiovascular Health Promotion. An issue that can no longer wait. J Am Coll Cardiol. 2018;72:908-913.

4. Moss HE, An Ruopeng, Nelson T, Kaigang Li. Risk of Atherosclerotic Cardiovascular Disease Among US Adults: Use of the 1999-2014 NHANES Data. J Primary Prevent. 2019;40:569-573.

5. Leifheit-Limson EC, D'Onofrio D, Daneshvar, M, et al. Sex Differences in Cardiac Risk Factors, Perceived Risk, and Health Care Provider Discussion of Risk and Risk Modification Among Young Patients with Acute Myocardial Infarction. J Am Coll Cardiol. 2015;66:1949-1957.

6. Tapia-Vierya JV, Delgado-Coello B, Mas Oliva J. Atherosclerosis and Cancer: A Resemblance with Farreaching Implications. Arch Med Res. 2017;48:12-26.

7. Weintraub WS, Daniels SR, Burke LE, et al. Value of Primordial and Primary Prevention for Cardiovascular Disease. A Policy Statement from the American Heart Association. Circulation. 2011;124:967-990.

8. Rosselo X, Dorresteijn J AN, Janssen A, et al. Risk prediction tools in cardiovascular disease prevention: A report from the ESC Prevention of CVD Programme Led by the European Association of Preventive Cardio- -logy (EAPC) in collaboration with the Acute Cardiovascular Association (ACCA), and the Association of Cardiovascular Nursing and Allied Health Professions (ACNAP). E Heart J: Acute Cardiovascular Care. 2019;26:1534-1544.

9. ASCVD Risk Estimator Plus. Accessed: January 28, 2019

10. Grundy SM, Stone NJ, Bailey AL, Beam C, Birtcher KK, et al. 2018 AHA/ACC/AACVPR/AAPA/ABC/ACPM/ADA/ AGS/APhA/ASPCN/NLA/PCNA. Guideline on the Management of Blood Cholesterol: Executive Summary. J Am Coll Cardiol. 2019;73:3168-3209.

11. 2019 European Heart Score. http://heartscore.org. Accessed: August 15, 2019

12. Gaziano JM, Brotons C, Coppolecchia R, et al. Use of aspirin to reduce risk of initial vascular events in patients at moderate risk of cardiovascular disease (ARRIVE): a randomized, double-blind, placebo-controlled trial. Lancet. 2018;392:1036-1046.

13. Lowenstein A, Li S, Navar AM, et al. Does clinician-reported lipid guideline adoption translate to guideline-adherent care? An evaluation of the Patient and Provider Assessment of Lipid Management (PALM) registry. Am Heart J. 2018;200:118-124.

14. Akyea RK, Kai J, Quershi N, et al. Sub-optimal cholesterol response to initiation of statins and future risk of cardiovascular disease. Heart. 2019;105:975-891.

15. Khera AV, Won $\mathrm{H}-\mathrm{H}$, Peloso GM, et al. Diagnostic Yield and Clinical Utility of Sequencing Familial Hypercholesterolemia Genes in Patients With Severe Hypercholesterolemia. J Am Coll Cardiol. 2016;67:2578-2589.

16. Yusuf S, Bosch J, Dagenais G, et al. Cholesterol Lowering in Intermediate-Risk Persons without Cardiovascular Disease. N Engl J Med. 2016;374:2021-31.

17. Ridker PM, Danielson E, Fonseca FA, et al. Rosuvastatin to Prevent Vascular Events in Men and Women with Elevated C-Reactive Protein. N Engl J Med. 2008;359:2127-2207.

18. Armanious MA, Mohammadi H, Khodor S, et al. Cardiovascular effects of radiation therapy. Curr Probl Cancer. 2018;42:433-442.

19. Lowell A, Suarez-Jimenez B, Helpman L, Zhu Z. 9/11-related PTSD among highly exposed populations: a systematic review 15 years after the attack. Psychol Med. 2018;48:537-553.

20. Lindgren P, Graff J, Olsson AG, et al. Costeffectiveness of high-dose atorvastatin compared with regular dose simvastatin. Eur Heart $\mathrm{J}$. 2007:28:1448-1453. 
21. Davies GM, Vyas A, Baxter CA. Economic evaluation of ezetimibe treatment in combination with statin therapy in the United States. J Med Econ. 2017;20:723-731.

22. Pandya A, Sy S, Cho S, et al. Cost-effectiveness of 10year Risk Thresholds for Initiation of Statin Therapy for Primary Prevention of Cardiovascular Disease. JAMA. 2015:314(2):142-150.

23. Kohli-Lynch CN, Bellows BK, Thanassoulis G, et al. Cost-effectiveness of Low-Density LipoproteinGuided Statin Treatment in Patients with Borderline Cardiovascular Risk. JAMA Cardiol. 2019;4:969-977.

24. van Bussel EF, Hoevenaar-Blom MP, Poortvliet RKE, et al. Predictive value of traditional risk factors for cardiovascular disease in older people: A systematic review. Prev Med. 2020;132:105986.

25. Cholesterol Treatment Trialist's Collaboration. Efficacy and safety of statin therapy in older people: a meta-analysis of individual participant data from 28 randomised controlled trials. Lancet. 2019;393:407415.

26. Eilat-Tsanani S, Mor E, Schonmann Y. Statin Use Over 65 years and All-Cause Mortality: A 10-year Follow-UP of 19,518 people. J Am Geriatr Soc. 2019;67:2038-2044.

27. Giral P, Neumann A, Weill A, Coste J. Cardiovascular effect of discontinuing statins for primary prevention at the age of 75 years: a nationwide population-based cohort study in France. Eur H Journal. 2019;40:35153525.

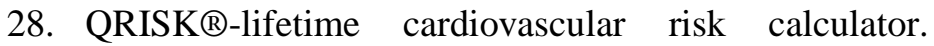
https://qrisk.org/lifetime/index.php/. Accessed: January 28, 2019.

29. DeFilippis AP, Young R, Carrubba CJ, et al. An Analysis of Calibration and Discrimination Among Multiple Cardiovascular Risk Scores in a Modern Multiethnic Cohort. Ann Intern Med. 2015;162:266275.

30. Greenland P, Blaha MJ, Budoff MJ, et al. Coronary Calcium Score and Cardiovascular Risk. J Am Coll Cardiol. 2018;72:434-447.

31. Mitchell JD, Fergerstrom N, Gage BF, et al. Impact of Statins on Cardiovascular Outcomes Following Coronary Artery Calcium Scoring. J Am Coll Cardiol. 2018;72:3233-3242.

32. Detrano R, Guerci AD, Carr JJ, et al. Coronary calcium as predictor of coronary events in four racial or ethnic groups. N Engl J Med. 2008;358:1336-1345.

33. Yeboah J, McClelland RL, Polonsky TS, et al. Comparison of novel risk markers for improvement in cardiovascular risk assessment in intermediate risk individuals. JAMA. 2012;308:788-795.

34. Stefanadis C, Antoniou C-K, Tsiachris D, Pietri P. Coronary Atherosclerotic Vulnerable Plaque: Current Perspectives. J Am Heart Assoc. 2017;6:e005543.

35. Iwasaki K, Matsumoto K. Relationship between coronary calcium score and high-risk plaque/signific- -ant stenosis. World J Cardiol. 2016; 8:48-487.

36. Blaha MJ, Silverman MG, Budoff MJ. Is There a Role for Coronary Artery Calcium Scoring for Management of Asymptomatic Patients at Risk for Coronary Artery Disease? Circulation: CV Imaging. 2014;7:398-408.

37. McLelland RL, Jorgensen NW, Budoff M, et al. 10-Year Coronary Heart Disease Risk with Validation in the Prediction Using Coronary Artery Calcium and Traditional Risk Factors: Derivation in the MESA (Multi-ethnic Study of Atherosclerosis HRN (Heinz Nixdorf Recall) Study and the DHS (Dallas Heart Study). J Am Coll Cardiol. 2015;66:1643-1653.

38. Chaikriangkrai K, Jhun HY, Sunkara A, et al. Coronary artery calcium score as a predictor for incident ischemic stroke: systematic review and meta-analysis. Int J Cardiol. 2017;236:473-477.

39. McNeil JJ, Woods RL, Nelson MR, et al. Effect of Aspirin on Disability-free Survival in the Healthy Elderly. N Engl J Med. 2018; 379:14991508.

40. ASCEND Study Collaborative Group. Effects of Aspirin for Primary Prevention in Persons with Diabetes Mellitus. N Engl J Med. 2018;379:1529-1539.

41. Zheng SL, Roddick AJ. Association of Aspirin Use for Primary Prevention With Cardiovascular Events and Bleeding Events. JAMA. 2019;321:277-287.

42. Chasman DI, Shiffman D, Zee RY, et al. Polymorphism in the apolipoprotein(a) gene, plasma lipoprotein(a), cardiovascular disease and low-dose aspirin therapy. Atherosclerosis. 2009;203:371-376.

43. Patnode CD, Evans CV, Senger CA, Redmond N, Lin JS. Behavioral Counseling to Promote a Healthful Diet and Physical Activity for Cardiovascular Disease Prevention in Adults Without Known Cardiovascular Disease Risk Factors: Updated Systematic Review for the US Preventive Services Task Force. JAMA 2017;318:175-193.

44. Lassale C, Gunter MJ, Romaguera D, et al. Diet Quality Scores and Prediction of All-Cause, Cardiovascular and Cancer Mortality in a PanEuropean Cohort Study. PLos One. 2016:11: e0159025.

45. Tapia-Vierya JV, Delgado-Coello B, Mas Oliva J. Atherosclerosis and Cancer: A Resemblance with Far-reaching Implications. Arch of Med Res. 2017;48:12-26.

46. Jha P, Ramasundarahettige $C$, Landsman $V$, et al. 21st-Century Hazards of Smoking and Benefits of Cessation in the United States. N Eng J Med. 2013;368:341-350. 
47. Patnode CD, Henderson JT, Thompson JH, et al. Behavioral Counseling and Pharmacotherapy Interventions for Tobacco Cessation in Adults, Including Pregnant Women: A Review of Reviews for the U.S. Preventive Services Task Force. Ann Int Med. 2015;163:608-621.

48. Lavie CJ, Laddu D, Arena R, et al. Healthy Weight and Obesity Prevention. J Am Coll Cardiol. 2018;72:1506-1531.

49. Kubzansky LD, Huffman JC, Boehm JK, et al. Positive Psychological Well-being and Cardiovascular Disease. J Am Coll Cardiol. 2018;72:1382-1396.

50. Vaccarino V, Badimon L, Bremmer JS, et al. Depression and coronary heart disease: 2018 ESC position paper of the working group of coronary pathophysiology and microcirculation developed under the auspices of the ESC Committee for Practice Guidelines. Eur Heart J. 2020;41:1687-1696.

51. Batty GD, Jung KJ, Mok Y, et al. Oral health and later coronary heart disease: cohort study of one million people. Eur J Prev Cardiol 2018;25:598-605.

52. Bradley SM, Michos ED, Miedema MD. Physical Activity, Fitness, and Cardiovascular Health. Editorial. JAMA Network Open. 2019;2:e198343.

53. Schwalm JD, McCready T, Lopez-Jaramillo P, et al. A community based comprehensive intervention to reduce cardiovascular risk in hypertension (HOPE-4): a cluster randomized controlled trial. Lancet 2019;394:1231-1242.

54. Whelton PK, Carey RM, Aronow WS, et al. 2017 ACC/AHA/AAPA/ABC/ACPM/AGS/APhA/ASH/AS PC/NMA/PCNA Guideline for the Prevention, Detection, Evaluation, and Management of High Blood Pressure in Adults. Hypertension. 2018;71:1299-2269.
55. Garber AJ, Abrahamson MJ, Barzilay JI, et al. Consensus Statement by the American Association of Clinical Endocrinologists and American College of Endocrinology on the Comprehensive Type 2 Diabetes Management Algorithm-2019 Executive Summary. Endocrine Pract. 2019;25:69100.

56. Rosenzweig JL, Bakris GL, Berglund LF, et al. Primary Prevention of ASCVD and T2DM in Patients at Metabolic Risk: An Endocrine Society Clinical Practice Clinical Practice Guideline. J Clin Endocrinol Metab. 2019;104:3939-3985.

57. Strelitz J, Ahern AL, Long GH, et al. Moderate weight change following diabetes diagnosis and 10year incidence of cardiovascular disease and mortality. Diabetologia. 2019;62:1391-1402.

58. Joseph MS, Konerman MA, Zhang M, et al. Longterm outcomes following completion of a structured nutrition and exercise lifestyle intervention program for patients with the metabolic syndrome. Diabetes Metab Syndr Obes. 2018;11:753-759.

59. Bhatt DL, Steg PG, Miller M, et al. Cardiovascular Risk Reduction by Icosapent Ethyl for Hypertriglyceridemia. N Engl J Med 2019;380:1122. 